\title{
Advances in Anatomic, Functional, and Molecular Imaging of Angiogenesis
}

Angiogenesis is a fundamental process in various physiologic and pathologic processes. The ability to visualize and quantify angiogenesis will allow early diagnosis and monitoring for clinical determination of angiogenesis states before, during, and after adjuvant antiangiogenic and therapeutic angiogenesis treatments.

Key Words: angiogenesis; volumetric computed tomography (VCT); dynamic contrast-enhanced magnetic resonance imaging (DCE-MRI); integrin; vascular endothelial growth factor receptor (VEGFR)

J Nucl Med 2008; 49:511-514

DOI: 10.2967/jnumed.107.050179

In recent years, angiogenesis has become one of the most important and intensely studied areas of cancer research, with the future holding great promise. Angiogenesis is a fundamental process in various physiologic and pathologic processes. The ability to visualize and quantify angiogenesis will allow early diagnosis and monitoring for clinical determination of angiogenesis states before, during, and after adjuvant antiangiogenic and therapeutic angiogenesis treatments. Investigators have identified more than 20 angiogenic growth factors, their receptors, and the details of their signal transduction pathways. Achievements with the vascular endothelial growth factor (VEGF) antibody bevacizumab (Avastin; Genentech) in combination with standard cytotoxic chemotherapy in metastasized colorectal cancer, breast cancer, and non-small cell lung cancer (1) are among them. However, although new antiangiogenic therapies are continually being introduced into clinical trials, we believe that the future success of these therapies will require the codevelopment of high-throughput, cost-effective imaging techniques with highly specific biomarkers of angiogenesis to determine optimal doses and individual response to therapy on a structural, functional, and molecular level. In the future, we believe that angiogenesis imaging will serve as an early diagnostic and monitoring tool for the clinical determination of angiogenesis states before, during, and after adjuvant antiangiogenic cancer therapy.

\section{STRUCTURAL IMAGING OF ANGIOGENESIS}

Several research and clinical imaging modalities are available for visualizing the structure of microvasculature such as intravital microscopy, CT angiography, contrast-enhanced ultrasound, and high-resolution magnetic resonance angiography. Multidetector

Received Jan. 31, 2008; revision accepted Mar. 6, 2008.

For correspondence or reprints contact: Xiaoyuan Chen, PhD, Molecular Imaging Program at Stanford (MIPS), Department of Radiology and Bio-X Program, Stanford University School of Medicine, 1201 Welch Rd., P095, Stanford, CA 94305-5484.

E-mail: shawchen@stanford.edu

COPYRIGHT @ 2008 by the Society of Nuclear Medicine, Inc.
CT angiography allows for larger scan volumes in less time with improved spatial and temporal resolution, making it possible to image different vascular phases using the same contrast bolus to compare the vasculature of tumors with that of normal organs. In addition, CT angiography provides a quick, noninvasive, low-dose alternative to conventional digital subtraction angiography in a research setting, and CT angiography has the potential to provide invaluable information about surrounding nonvascular structures and both arterial and venous systems. However, the spatial resolution of MDCT is still not enough for microvasculature visualization (2). To improve the number of photons produced per unit-area per second of benchtop x-ray sources, radiation micro-CT using synchrotron light has been applied for improved spatial and contrast resolution. Several cubic millimeters of microvasculature including capillaries can be visualized with a combination of pure absorption and edge enhancement at a voxel size of $1.4 \mu \mathrm{m}$ (3).

Micro-CT is a promising method to visualize the vascular architecture of normal organs and tumors and to derive quantitative imaging information. However, the long scan periods and high x-ray doses of micro-CT make it unsuitable for repeated noninvasive vessel measurements. Kiessling et al. reported a prototype volumetric CT apparatus for high-resolution imaging in experimental and preclinical applications in vivo that combines the advantages of micro-CT and clinical CT scanners (4). Reconstructed volumetric CT images have a high resolution, with isotropic voxels of less than $4 \times 10^{-4} \mathrm{~mm}^{3}$, and the minimum vessel diameter visualized by volumetric CT varies between 40 and $50 \mu \mathrm{m}$ (Fig. 1). Advances with volumetric CT help fill the gap between clinical multislice CT and preclinical micro-CT systems and is highly suited for studying orthotopic and metastasizing tumor models.

\section{FUNCTIONAL IMAGING OF ANGIOGENESIS}

Dynamic contrast-enhanced MRI is a noninvasive method of imaging microvascular structure by tracking the pharmacokinetics of an injected contrast agent as it passes through tumor vasculature. This technique measures changes in vascular permeability, extravascular and vascular volumes, and blood flow. Dynamic contrastenhanced MRI has been used for evaluation of drug efficacy in several trials of antiangiogenic drugs. Overall, dynamic contrastenhanced MRI is promising as an imaging biomarker in clinical trials. However, data on its performance, especially for response assessment, are not uniform and seem to depend strongly on the therapy protocol and tumor type.

The simplicity, ease of use, speed, and safety of ultrasound for angiogenesis imaging has led to its increased use in disease diagnosis, treatment assessment, follow-up, and therapy guidance. Ultrasound can be applied to image the microcirculation using both Doppler and microbubble techniques. Power Doppler can be quantified to give an estimate of relative fractional vascular volume and blood velocity. Innovations in the field of ultrasound imaging 
FIGURE 1. (A) Three-dimensional visualization showing volumetric CT angiography of tumor-bearing nude mouse, and detailed image of tumor after magnification (inset). Dilatation of subcutaneous vessels (arrow on whole-body visualization) that interact with tumor tissue is clearly visible. (B) On 3-dimensional volume rendering, larger vessels are seen inside tumor. Determined vessel diameters were $145 \mu \mathrm{m}$ (1) and $205 \mu \mathrm{m}$ (2). (Reprinted with permission of (4).)
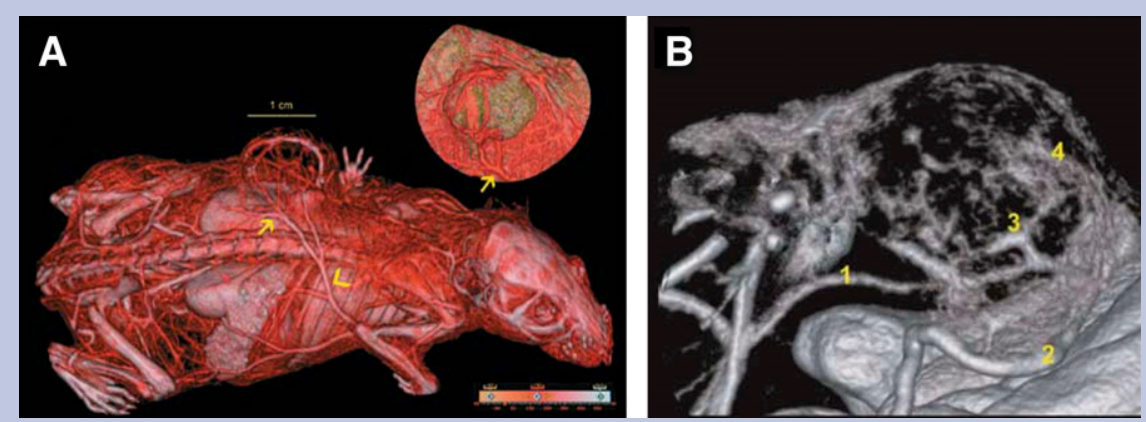

have made it possible to measure blood-flow microvasculature of less than $100 \mu \mathrm{m}$. At frequencies on the order of $50 \mathrm{MHz}$, Doppler processing allows for the direct assessment of flow dynamics in real time in arterioles as small as $15 \mu \mathrm{m}$ (5). Ultrasound using microbubbles can show blood flow down to the microcirculation level by raising the signal emitted from smaller vessels. However, microbubbles are limited by their relatively larger size $(1-10 \mu \mathrm{m}$ in diameter). This increased size in comparison with smaller contrast agents provides access to the bulk properties of the microvascular compartment but does not provide sufficient resolution to allow observation of microvasculature morphology and detailed flow characteristics.

Optical imaging is a low-cost method that offers single-cell resolution and real-time imaging to monitor tumor-host interactions - a component critical to tumor development and angiogenesis. Optical imaging has the ability to determine tumor vascular permeability, vessel size, and blood flow in vivo and can offer great clarity for imaging blood vessels using fluorescent dyes or quantum dots. Several high-resolution microscopic optical imaging techniques have recently been developed to study molecular events in vivo such as intravital fluorescence microscopy, confocal laser scanning microscopy, multiphoton laser scanning microscopy, and in situ scanning force microscopy $(6,7)$. The major limitation of optical imaging is tissue light scattering and absorption, which affect both image resolution and the depth of light penetration in tissues.

\section{MOLECULAR IMAGING}

Although imaging techniques such as dynamic contrast-enhanced MRI are widely used to assess hemodynamic parameters, the physiologic meaning of results from these studies often remains difficult to interpret. Therefore, more specific markers of tumor angiogenic activity are necessary for pretherapeutic angiogenesis assessment and posttherapy response evaluation. Molecules regulating angiogenesis include, but are not limited to, growth factor receptors, tyrosine kinase receptors, G-protein-coupled receptors for angiogenesis modulating proteins, integrins, and matrix metalloproteinases. Here, we will focus on 2 of the most intensively studied angiogenesis-related molecular targets: integrin $\alpha_{v} \beta_{3}$ and VEGF receptors (VEGFRs).

Integrins are a family of cell adhesion molecules playing key roles during tumor angiogenesis and metastasis $(8,9)$. Integrins expressed on endothelial cells modulate cell migration and survival during tumor angiogenesis, whereas integrins expressed on carcinoma cells potentiate metastasis by facilitating invasion and movement across blood vessels. The $\alpha_{\mathrm{v}} \beta_{3}$ integrin, which binds to arginine-glycineaspartic acid (RGD)-containing components of the extracellular matrix, is significantly upregulated on tumor vasculature but not on quiescent endothelium $(9,10)$. Several comprehensive reviews on multimodality imaging of integrin expression (PET, SPECT, MRI, ultrasound, and optical) using appropriate cyclic RGD peptides and monoclonal antibodies against integrin $\alpha_{v} \beta_{3}$ have been published (11-16). Among these, PET of integrin $\alpha_{v} \beta_{3}$ using the radiotracer ${ }^{18} \mathrm{~F}-$ galacto-RGD has been tested in humans with good contrast and with a low radiation dose, comparable to that of ${ }^{18}$ F-FDG (Fig. 2) (17-20). It has also been shown that the intensity of ${ }^{18} \mathrm{~F}$-galacto-RGD uptake correlates with $\alpha_{\mathrm{v}} \beta_{3}$ expression in murine tumor xenografts and in patients with cancer. The same tracer is now being evaluated for clinical use in different tumor entities.

Most monomeric RGD peptide-based tracers tend to have fast blood clearance accompanied by relatively low tumor uptake and rapid tumor washout, presumably because of the suboptimal receptor-binding affinity/selectivity and inadequate contact with

FIGURE 2. Comparison of different uptake patterns in ${ }^{18} \mathrm{~F}-\mathrm{FDG}$ PET and ${ }^{18} \mathrm{~F}$ galacto-RGD PET. (A) Patient with nonsmall cell lung cancer of left upper lobe (small arrow) and multiple metastases to bone (large arrow), liver, lymph nodes, and adrenal glands. Uptake in ${ }^{18} \mathrm{~F}-\mathrm{FDG}$ PET image is intense, whereas uptake in ${ }^{18} \mathrm{~F}$ galacto-RGD PET image is substantially lower. (B) Patient with neuroendocrine tumor of bronchus in right lower lobe (small arrow) and multiple metastases to bone (large arrows), liver, spleen, and lymph nodes. This patient shows more intense uptake in lesions on ${ }^{18} \mathrm{~F}$-galacto-
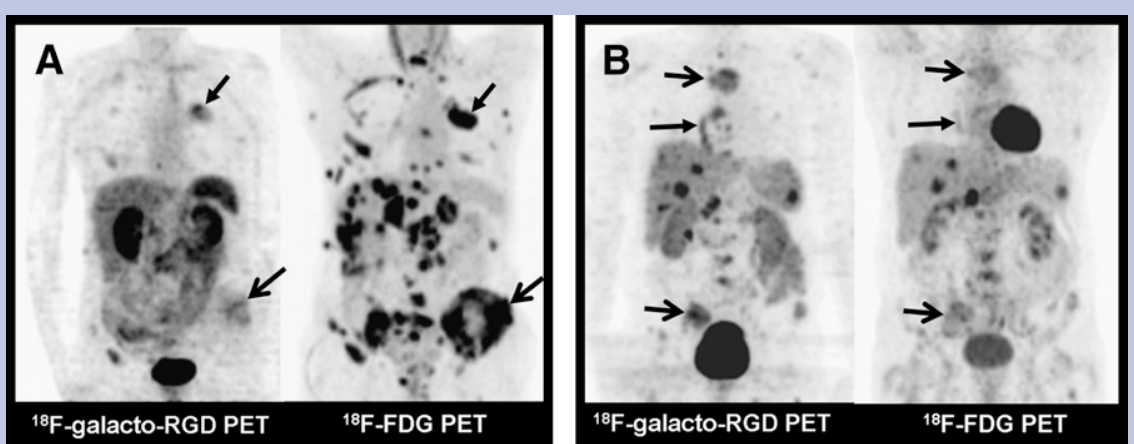
RGD PET than on ${ }^{18} \mathrm{~F}-\mathrm{FDG}$ PET. 
the binding pocket in the extracellular segment of integrin $\alpha_{\mathrm{v}} \beta_{3}$. Multimerization of cyclic RGD peptides has been repeatedly demonstrated to increase integrin affinity and enhance antiadhesion ability against integrin $\alpha_{\mathrm{v}} \beta_{3}$ and thus significantly improve tumor targeting over the monomeric RGD analogs (11,12,21-26). The polyvalency effect has been most beneficial in tumors with low to medium integrin expression (26). Several dimeric and multimeric RGD peptide-based imaging probes are in the process of clinical translation for the first studies on humans. Whether such new probes will have advantages over ${ }^{18} \mathrm{~F}$-galacto-RGD remains to be elucidated.

Integrin $\alpha_{v} \beta_{3}$ is not an angiogenesis marker per se because of its nonexclusive expression on the neovasculature. Small molecules, even antibody-based probes for integrin expression, do not provide an accurate readout of tumor angiogenesis because these probes bind to integrin $\alpha_{\mathrm{v}} \beta_{3}$ expressed on both the tumor vasculature and tumor cells. Because of their relatively large sizes and rigid structure, integrin-targeted nanoparticles that do not extravasate are probably true vascular integrin-specific probes $(27,28)$. A few such examples include RGD peptide-coupled quantum dots for near-infrared fluorescence imaging, single-walled nanotubes for Raman and photoacoustic imaging, liposomal and perfluorocarbon nanoparticles for T1-weighted MRI, ultrasmall superparamagnetic iron oxide particles for T2-weighted MRI, and microbubbles for contrast-enhanced ultrasound. Several of these nanoparticle conjugates have also been suitably labeled for radionuclide imaging to assess the tumortargeting efficacy and in vivo kinetics of the particles in a more quantitative manner.

The angiogenic actions of VEGF are mediated mainly via 2 endothelium-specific receptor tyrosine kinase receptors, Flt-1 (VEGFR-1) and Flk-1/KDR (VEGFR-2). Both VEGFRs are restricted largely to vascular endothelial cells, and all VEGF-A isoforms bind to both VEGFR-1 and VEGFR-2. It is now generally accepted that VEGFR-2 is the major mediator of the mitogenic, angiogenic, and permeability-enhancing effects of VEGF. The critical role of VEGF-A in cancer progression has been highlighted by the approval of the humanized anti-VEGF monoclonal antibody bevacizumab (Avastin; Genentech) for first-line treatment (29). The flourishing investigations of both radionuclide- and non-radionuclidebased VEGFR imaging studies stem from the successful initial clinical evaluation of ${ }^{123} \mathrm{I}_{-} \mathrm{VEGF}_{165}$ in patients with gastrointestinal cancer $(30,31)$. Development of VEGF- or VEGFR-targeted molecular imaging probes is a potential new paradigm for assessing antiangiogenic therapies and clarifying the role and expression profile of VEGF/VEGFR in angiogenesis-related diseases (32).

Imaging probes based on wild-type VEGF-A isoforms bind to both VEGFR-1 and VEGFR-2. The kidneys have high VEGFR1 expression that can take up VEGF-A-based tracer and thus usually makes the kidney the dose-limiting organ $(33,34)$. Because VEGFR-2 is generally accepted to be more functionally important than VEGFR-1 in cancer progression, the ability to image VEGFR-2 expression can be a valuable tool for evaluating patients with a variety of malignancies, particularly those undergoing antiVEGFR-2 therapies. Alanine-scanning mutagenesis has been used to identify a positively charged surface in $\mathrm{VEGF}_{165}$ that mediates binding to VEGFR-2 (35). $\operatorname{Arg}_{82}, \mathrm{Lys}_{84}$, and His ${ }_{86}$, located in a hairpin loop, were found to be critical for binding VEGFR-2, whereas negatively charged residues, $\mathrm{Asp}_{63}, \mathrm{Glu}_{64}$, and $\mathrm{Glu}_{67}$, were associated with VEGFR-1 binding. A VEGFR-2-specific PET tracer has thus been developed using the D63AE64AE67A mutant of $\mathrm{VEGF}_{121}\left(\mathrm{VEGF}_{\mathrm{DEE}}\right)$ generated by recombinant DNA technology. Renal uptake of ${ }^{64} \mathrm{Cu}$-tetraazacyclododecanetetraacetic acid

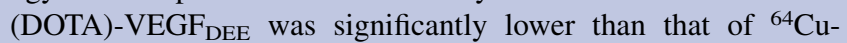
DOTA-VEGF $_{121}$ (Fig. 3) (36). Further improvement in VEGFR-2 binding affinity/specificity, pharmacokinetics, and tumor-targeting efficacy by generation of other VEGF $_{121}$ mutants is considered a good direction for clinical translation of VEGF protein-based imaging probes.

\section{FUTURE PERSPECTIVES}

Numerous imaging techniques are now available to visualize tissue vasculature on a structural, functional, and molecular level. All these techniques have undergone significant preclinical development in
A

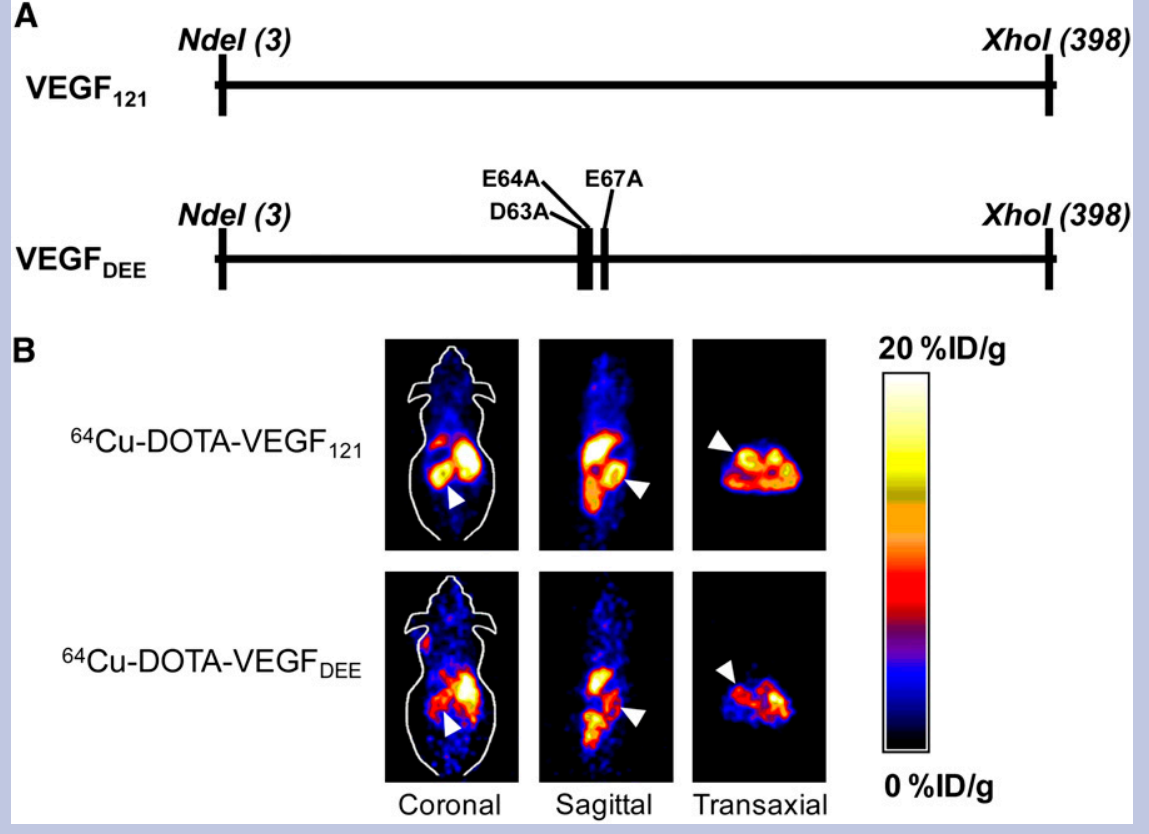

FIGURE 3. (A) Schematic representation of VEGF 121 and its VEGFR-2-specific mutant VEGF $F_{\mathrm{DEE}}$ (mutated at 63, 64, and 67 positions). (B) Coronal, sagittal, and axial small-animal PET images containing kidney (arrowheads) at $4 \mathrm{~h}$ after injection of ${ }^{64} \mathrm{Cu}$-DOTA-VEGF 121 or ${ }^{64} \mathrm{Cu}$-DOTA$V_{E G F} F_{D E E}$. Ablation of VEGFR-1 binding is most responsible for reduced kidney uptake of radiolabeled VEGF mutant. (Reprinted with permission of (36).) 
recent years and should aid in future assessment of angiogenesis, monitoring of antiangiogenic treatment, and antiangiogenic drug development. Only vascular imaging of functional hemodynamic parameters such as $\mathrm{K}^{\text {trans }}$, blood flow, and blood volume are currently being used clinically to assess antiangiogenic and cytotoxic chemotherapies. However, the physiologic meaning of these results is often difficult to interpret.

With molecular imaging of angiogenesis, only a few radiotracers have been used in humans thus far, and the role of these tracers in assessing response to antiangiogenic therapies is still undetermined. Although integrin $\alpha_{v} \beta_{3}$ is by far the most extensively studied angiogenic factor for imaging, future trials still need to elucidate which target structure is optimal for assessing angiogenesis. In regard to which imaging technique is optimal, the radiotracer approach will likely be the first to be used on a wider scale in patients in the intermediate term because of its high sensitivity and the low amounts of tracer required. However, over the long term, MRI might be a better imaging alternative because of its lack of ionizing radiation and high spatial resolution. Overall, it is likely that angiogenesis will eventually be assessed not using a single parameter, target structure, or imaging technique but rather a combination of parameters that allow for a multimodal imaging evaluation of the intricacies of the angiogenic cascade. Assessing the different aspects of angiogenesis at the structural, functional, and molecular levels for clinical determination of angiogenesis states before, during, and after antiangiogenic therapy or therapeutic angiogenesis will likely become a reality and help further steps toward personalized medicine.

\section{Andrew R. Hsu Xiaoyuan Chen Stanford University School of Medicine Stanford, California}

\section{REFERENCES}

1. Hurwitz H, Fehrenbacher L, Novotny W, et al. Bevacizumab plus irinotecan, fluorouracil, and leucovorin for metastatic colorectal cancer. $N$ Engl J Med. 2004;350:2335-2342.

2. Engelke C, Schaefer-Prokop C, Schirg E, Freihorst J, Grubnic S, Prokop M. High-resolution CT and CT angiography of peripheral pulmonary vascular disorders. Radiographics. 2002;22:739-764.

3. Plouraboue F, Cloetens P, Fonta C, Steyer A, Lauwers F, Marc-Vergnes JP. X-ray high-resolution vascular network imaging. J Microsc. 2004;215:139-148.

4. Kiessling F, Greschus S, Lichy MP, et al. Volumetric computed tomography (VCT): a new technology for noninvasive, high-resolution monitoring of tumor angiogenesis. Nat Med. 2004;10:1133-1138.

5. Ferrara KW, Merritt CR, Burns PN, Foster FS, Mattrey RF, Wickline SA. Evaluation of tumor angiogenesis with US: imaging, Doppler, and contrast agents. Acad Radiol. 2000;7:824-839.

6. Brown EB, Campbell RB, Tsuzuki Y, et al. In vivo measurement of gene expression, angiogenesis and physiological function in tumors using multiphoton laser scanning microscopy. Nat Med. 2001;7:864-868.

7. Jain RK, Munn LL, Fukumura D. Dissecting tumour pathophysiology using intravital microscopy. Nat Rev Cancer. 2002;2:266-276.

8. Hynes RO. Integrins: bidirectional, allosteric signaling machines. Cell. 2002; 110:673-687.

9. Hood JD, Cheresh DA. Role of integrins in cell invasion and migration. Nat Rev Cancer. 2002;2:91-100.

10. Xiong JP, Stehle T, Zhang R, et al. Crystal structure of the extracellular segment of integrin alpha Vbeta3 in complex with an Arg-Gly-Asp ligand. Science. 2002;296:151-155.
11. Cai W, Rao J, Gambhir SS, Chen X. How molecular imaging is speeding up antiangiogenic drug development. Mol Cancer Ther. 2006;5:2624-2633.

12. Cai W, Gambhir SS, Chen X. Multimodality tumor imaging targeting integrin $\alpha_{\mathrm{v}} \beta_{3}$. Biotechniques. 2005;39(suppl):S6-S17.

13. Liu S. Radiolabeled multimeric cyclic RGD peptides as integrin alphavbeta 3 targeted radiotracers for tumor imaging. Mol Pharm. 2006;3:472-487.

14. Haubner R. Alpha beta $_{3}$-integrin imaging: a new approach to characterise angiogenesis? Eur J Nucl Med Mol Imaging. 2006;33(suppl 1):54-63.

15. Choe YS, Lee KH. Targeted in vivo imaging of angiogenesis: present status and perspectives. Curr Pharm Des. 2007;13:17-31.

16. Chen X. Multimodality imaging of tumor integrin alphavbeta3 expression. Mini Rev Med Chem. 2006;6:227-234.

17. Haubner R, Weber WA, Beer AJ, et al. Noninvasive visualization of the activated alphavbeta3 integrin in cancer patients by positron emission tomography and [ ${ }^{18}$ F]galacto-RGD. PLoS Med. 2005;2:e70.

18. Beer AJ, Niemeyer M, Carlsen J, et al. Patterns of $\alpha_{\mathrm{v}} \beta_{3}$ expression in primary and metastatic human breast cancer as shown by ${ }^{18} \mathrm{~F}$-galacto-RGD PET. $J$ Nucl Med. 2008;49:255-259.

19. Beer AJ, Haubner R, Wolf I, et al. PET-based human dosimetry of ${ }^{18} \mathrm{~F}$-galactoRGD, a new radiotracer for imaging alpha v beta3 expression. J Nucl Med. 2006; 47:763-769.

20. Beer AJ, Lorenzen S, Metz S, et al. Comparison of integrin alphaVbeta3 expression and glucose metabolism in primary and metastatic lesions in cancer patients: a PET study using ${ }^{18}$ F-galacto-RGD and ${ }^{18}$ F-FDG. J Nucl Med. 2008; 49:22-29.

21. Chen X, Liu S, Hou Y, et al. MicroPET imaging of breast cancer $\alpha_{\mathrm{v}}$-integrin expression with ${ }^{64} \mathrm{Cu}$-labeled dimeric RGD peptides. Mol Imaging Biol. 2004; 6:350-359.

22. Patterson BD, Abela R, Auderset H, et al. The materials science beamline at the Swiss Light Source: design and realization. Nucl Instrum Methods Phys Res A. 2005;540:42-67.

23. Chen X, Tohme M, Park R, Hou Y, Bading JR, Conti PS. Micro-PET imaging of $\alpha_{\mathrm{v}} \beta_{3}$-integrin expression with ${ }^{18} \mathrm{~F}$-labeled dimeric RGD peptide. Mol Imaging. 2004;3:96-104.

24. Cacciari B, Spalluto G. Non peptidic $\alpha_{v} \beta_{3}$ antagonists: recent developments. Curr Med Chem. 2005;12:51-70.

25. Kennedy JE, ter Haar GR, Wu F, et al. Contrast-enhanced ultrasound assessment of tissue response to high-intensity focused ultrasound. Ultrasound Med Biol. 2004;30:851-854.

26. Li ZB, Cai W, Cao Q, et al. ${ }^{64} \mathrm{Cu}$-labeled tetrameric and octameric RGD peptides for small-animal PET of tumor $\alpha_{v} \beta_{3}$ integrin expression. J Nucl Med. 2007; 48:1162-1171.

27. Cai W, Chen X. Multimodality molecular imaging of tumor angiogenesis. J Nucl Med. In press.

28. Cai W, Chen X. Nanoplatforms for targeted molecular imaging in living subjects. Small. 2007;3:1840-1854.

29. Ferrara N, Hillan KJ, Gerber HP, Novotny W. Discovery and development of bevacizumab, an anti-VEGF antibody for treating cancer. Nat Rev Drug Discov. 2004;3:391-400.

30. Li S, Peck-Radosavljevic M, Kienast $\mathrm{O}$, et al. Imaging gastrointestinal tumours using vascular endothelial growth factor-165 $\left(\mathrm{VEGF}_{165}\right)$ receptor scintigraphy. Ann Oncol. 2003;14:1274-1277.

31. Li S, Peck-Radosavljevic M, Kienast O, et al. Iodine-123-vascular endothelial growth factor-165 ( $\left.{ }^{123} \mathrm{I}_{-} \mathrm{VEGF}_{165}\right)$ : biodistribution, safety and radiation dosimetry in patients with pancreatic carcinoma. Q J Nucl Med Mol Imaging. 2004;48: 198-206.

32. Cai W, Chen X. Multimodality imaging of vascular endothelial growth factor and vascular endothelial growth factor receptor expression. Front Biosci. 2007;12: 4267-4279.

33. Cai W, Chen K, Mohamedali KA, et al. PET of vascular endothelial growth factor receptor expression. J Nucl Med. 2006;47:2048-2056.

34. Backer MV, Levashova Z, Patel V, et al. Molecular imaging of VEGF receptors in angiogenic vasculature with single-chain VEGF-based probes. Nat Med. 2007;13:504-509.

35. Keyt BA, Nguyen HV, Berleau LT, et al. Identification of vascular endothelial growth factor determinants for binding KDR and FLT-1 receptors: generation of receptor-selective VEGF variants by site-directed mutagenesis. $J$ Biol Chem. 1996;271:5638-5646.

36. Wang H, Cai W, Chen K, et al. A new PET tracer specific for vascular endothelial growth factor receptor 2. Eur J Nucl Med Mol Imaging. 2007;34: 2001-2010. 